\title{
Pacific
}

Journal of

Mathematics

\section{FACTORIZATION METHOD FOR A BIMEROMORPHIC} MORPHISM

\author{
Jose PeRez Blanco
}




\title{
FACTORIZATION METHOD FOR A BIMEROMORPHIC MORPHISM
}

\section{Jose Perez Blanco}

\begin{abstract}
The main goal of this paper is to develop a constructive factorization method for a bimeromorphic morphism between smooth germs of complex analytic spaces of dimension 3 provided is composition of blowing- ups with smooth, closed and irreductible centers. The factorization is determined by the irreductible components of the morphism's exceptional divisor. This result may be used for instance to prove the invariance of the characteristic pairs of quasi-ordinary singularities.
\end{abstract}

1. Notation and terminology. In the following $Z$ will be a smooth complex analytic space of dimension $3, \pi=\pi_{1} \circ \ldots \circ \pi_{n}$ : $Z(n) \rightarrow Z$ will be a chain of blowing-ups, $E(i, i)$ the exceptional divisor of each $\pi_{i} ; E(i, j)$ the strict transform of $E(i, i)$ by $\pi_{\imath+1} \circ$ $\ldots \circ \pi_{j}$, and $E_{\imath}=E(i, n)$.

Let $S$ be a surface in $Z$, let us call $S_{i}$ to the strict transform of $S_{\imath-1}$ by $\pi_{2}$ and $m(S, i)$ to the multiplicity of $S_{\imath-1}$ along the center of $\pi_{\imath}$. If $D$ is a divisor of $Z$, then $D^{t}$ will be the pull back of $D$ by $\pi$.

We will call a local blowing-up of a analytic germ $(Z, \mathbf{p})$ to the morphism

$$
(\pi):\left(Z^{\prime}, \pi^{-1}(\mathbf{p})\right) \rightarrow(Z, \mathbf{p})
$$

induced over the germ of $Z^{\prime}$ along the closed analytic: subspace $\pi^{-1}(\mathbf{p})$ by a composition $\pi: Z^{\prime} \rightarrow Z$ of blowing-ups.

A good local blowing-up will be a local blowing-up $(\pi)=\left(\pi_{1} \circ\right.$ $\left.\ldots \circ \pi_{n}\right)$ such that for all $i$ the center of $\pi_{\imath}$ is an irreducible smooth and closed subspace with normal crossings with $E(j, i), \forall j<i$.

Two blowing-up's chains $\pi=\pi_{1} \circ \ldots \circ \pi_{n}: Z^{\prime} \rightarrow Z, \delta=\delta_{1} \circ \ldots \circ \delta_{n}$ : $T \rightarrow Z$ are called equivalent if does exist a permutation $\sigma$, and $\beta: Z^{\prime} \rightarrow T$ in such a way that $\pi=\beta \circ \delta$, and $\beta\left(E_{\imath}\right)=T_{\sigma(\imath)}$, being $E_{1}+\ldots+E_{n}, T_{1}+\ldots+T_{n}$ the exceptional divisors of $\pi$ and $\delta$. Two local blowing-up $(\pi),(\delta)$ of the germ $(Z, \mathbf{p})$ are equivalent if they have equivalent representatives $\pi, \delta$. 


\section{Quadratic transformations.}

LEMMA 2.1. Let $\pi_{1} \circ \ldots \circ \pi_{n}: Z^{\prime} \rightarrow Z$ be a chain of blowing-up with irreductible centers. If for each $j \neq i$ is $\pi\left(E_{j}\right) \neq \pi\left(E_{i}\right)$ and if $\pi^{-1}\left(\pi\left(E_{n}\right)\right)$ is a divisor of $Z^{\prime}$, then $\pi$ is equivalent to another chain whose first transformation is the blowing-up with center $\pi\left(E_{n}\right)$.

Proof. Follows easily from the commutativity of the factors as a consequence of the hypothesis.

Let $D=\sum_{i \in I} m_{i} E_{i}$ be a divisor of $Z\left(m_{i} \neq 0\right.$ and $E_{i} \neq E_{j}$ if $i \neq j$ ), $D$ will be called $A$-divisor of $Z$ if no non zero divisor $\sum_{i \in I} n_{i} E_{i}$ is principal. For instance the exceptional divisor of a chain of blowing-up is an $A$-divisor.

Proposition 2.2. Let $S$ be a surface of $Z, Y$ a closed subspace of $Z, D_{Z} S$ the associated divisor to $S$, and $m$ the multiplicity of $S$ along $Y$. If $D_{Z}$ is linearly equivalent to an A-divisor $D, \pi: Z(1) \rightarrow$ $Z$ is the blowing-up of $Z$ with center $Y$, and $E$ is the exceptional divisor of $\pi$, then $D^{t}-m E$ is an A-divisor and is linearly equivalent to $D_{Z(1)} S_{1}$.

Proof. If $D$ is an $A$-divisor then $D^{t}$ is an $A$-divisor, because the property of being principal for a divisor is stable by blowing-up's pull back, then being $D-D_{Z} S$ principal because $D$ and $D_{Z} S$ are equivalent, $\left(D-D_{Z} S\right)^{t}$ is principal, and:

$$
\left(D-D_{Z} S\right)^{t}=D^{t}-\left(D_{Z} S\right)^{t}=\left(D^{t}-m E\right)-D_{Z(1)} S_{1}
$$

then $D_{Z(1)} S_{1}$ and $D^{t}-m E$ are equivalent.

We will call tree of the chain of blowing-up $\pi=\pi_{1} \circ \ldots \circ \pi_{n}$ to the set $\left\{E_{\imath}: 1 \leq i \leq n\right\}$ together with the arrow $\left(E_{i}, E_{j}\right)$ for all $(i, j)$ such that the center of $\pi_{j}$ is contained in $E(i, j-1)$. We will call ordered tree of $\pi$ to the tree of $\pi$ jointly with the weight $i$ in each $E_{i}$.

Theorem 2.3. Let $\pi=\pi_{1} \circ \ldots \circ \pi_{n}: Z^{\prime} \rightarrow Z$ be a chain of blouing-ups, $S$ a surface of $Z$, and $D$ an $A$-divisor. If $D$ and $D_{Z} S$ are equivalent then the A-divisor:

$$
D^{\prime}-\sum_{1 \leq i \leq n} a_{l} E_{1}-\left[m(S, n)+\sum_{1 \leq i \leq n} a_{i} m(E(i, n-1), n)\right] E_{n}
$$


is equivalent to $D_{Z(n)} S_{n}$.

Proof. We are going to prove the theorem by induction over $n$.

If $n=1$ the theorem is Proposition 2.2.

Let $n>1$ and let us suppose that the asertion is true until $n-1$, therefore there is an $A$-divisor $\left(D_{n-1}\right)^{t}-\sum_{1 \leq i \leq n-1} a_{i} E(i, n-1)$, equivalent to $D_{Z(n-1)} S_{n-1}$, then by Prop. 2.2 the divisor $\left[\left(D_{n-1}\right)^{t}-\right.$ $\left.\sum_{1 \leq i \leq n-1} a_{i} E(i, n-1)\right]^{t}-m E$, and $D_{Z(n)} S_{n}$ are equivalent. We conclude the proof because

$$
[E(i, n-1)]^{t}=E_{i}+m[E(i, n-1), n] E_{n} .
$$

With the above notation we will say that

$$
\sum_{1 \leq i \leq n} a_{i} E_{i}=D_{Z(n)} \pi^{-1}(S)-D_{Z(n)} S_{n}
$$

is the divisor associated to $S$ by $\pi$.

COROLlary 2.4. Let $\pi: Z^{\prime} \rightarrow Z$ a chain of $n$ quadratic transformations whose centers are contained in the sucesive strict transform of a surface $S$. Let $\sum_{1 \leq i \leq n} a_{i} E_{i}$, the $A$-divisor associated to $S$ by $\pi$, with the $\pi_{i}$ 's ordered in such a way that $a_{1} \leq \ldots \leq a_{n}$, then there is a chain of quadratic transformation $\delta_{1} \circ \ldots \circ \delta_{n}$ equivalent to $\pi$ such that for each $E_{i}=E(i, n), E(i, i)$ is the exeptional divisor of $\delta_{\imath}$.

Proof. Being all the transformations quadratic, for each pair of components of $\pi$ there are two possibilities:

(a) The center of the first one is contained in the center of the second.

(b) We are in the situation of Lemma 2.1.

In case (a) the inequality follows, and in case (b) by Lemma 2.1 the factor's order don't make any difference.

3. Combinatorial blowing-up. A chain of blowing-up $\pi=$ $\pi_{1} \circ \ldots \circ \pi_{n}: Z(n) \rightarrow Z$ is said to be a combinatorial blowingup if there are smooth and irreductible surfaces $\{X, Y, T\}$ of $Z$, with normal crossings, in such a way that the center of every component $\pi_{2+1}$ of $\pi$ is the intersection of some surfaces of the family 
$\left\{X_{i}, Y_{i}, T_{i}, E(i, j): 1 \leq j \leq i\right\}$. In the case under consideration we will say that $\{X, Y, T\}$ are the surfaces associated to $\pi$.

A combinatorial blowing-up $\pi$ will be called special if for every $i$, $1 \leq i \leq n$, the center of $\pi_{i+1}$ is contained in $E(i, i)$.

THEOREM 3.1. Given a special combinatorial blowing-up $\pi$ there is an algorithm to decide the tree of $\pi$.

Proof. Let $\{X, Y, T\}$ surfaces associated to $\pi$, and $\sum_{i \geq 1} a_{i, 1} E_{\imath}$, $\sum_{i \geq 1} b_{i, 1} E_{i}, \sum_{i \geq 1} c_{i, 1} E_{i}$, respectively the associated divisors to $X, Y$, $T$ by $\pi$.

In view of Theorem $2.3 E_{i}$ is the exceptional divisor of $\pi_{1}$ if and only if for all $j$ is :

$$
a_{i, 1} \leq a_{j, 1}, b_{i, 1} \leq b_{j, 1}, c_{i, 1} \leq c_{j, 1}
$$

Let $E_{1}$ the exceptional divisor of $\pi_{1}$, then there are two posibilities:

(a) If $a_{1,1}+b_{1,1}+c_{1,1}=3$ then $\pi_{1}$ is a quadratic transformation.

(b) If $a_{1,1}+b_{1,1}+c_{1,1}=2$ then $\pi_{1}$ is a monoidal transformation.

Moreover if $a_{1,1}=1$ (resp. $b_{1,1}, c_{1,1}=1$ ) the center of $\pi_{1}$ is contained in $X$ (resp. in $Y, T)$.

There is at least one of these surfaces which contained the center of $\pi_{1}$ and the center of $\pi_{2}$ is not contained in the your strict transform (this one is $X$ if $a_{1,1}=1$ and $b_{1,1}=1$ (resp. $c_{1,1}=1$ ) then for all $i$ is $a_{i, 1} \leq b_{i, 1}$ (resp. $\left.a_{i, 1} \leq c_{i, 1}\right)$ ) in this situation the associated divisors by $\pi_{2} \circ \ldots \circ \pi_{n}$ to $E(1,1), Y_{1}, T_{1}$ respectively are:

$$
\begin{aligned}
\sum_{i \geq 2} a_{i, 2} E_{i} & =\sum_{i \geq 2} a_{i, 1} E_{i} \\
\sum_{i \geq 2} b_{i, 2} E_{i} & =\sum_{i \geq 2} b_{i, 1} E_{i}-b_{1,1} \sum_{i \geq 2} a_{i, 1} E_{i} \\
\sum_{i \geq 2} c_{i, 2} E_{i} & =\sum_{i \geq 2} c_{i, 1} E_{i}-c_{1,1} \sum_{i \geq 2} a_{i, 1} E_{i} .
\end{aligned}
$$

By repeating this process with these divisors instead of the trivial ones we get the desired construction.

Let us write $a(i, j)=1$ when $\left(E_{i}, E_{\jmath}\right)$ is an arrow of the ordered tree of a chain of blowing-ups $\pi$, and $a(i, j)=0$ if that does not happen. 
By Theorem 2.3 the associated divisor to $S$ by $\pi$ is :

$$
D_{Z} \pi^{-1}(S)-D_{Z} S_{n}=\sum_{1 \leq i \leq n} c_{\imath} E_{i}
$$

with $c_{i}=m(S, i)+\sum_{\jmath<i} c_{j} a(j, i)$.

Then we have $m(S, 1)=$ multiplicity of $\pi^{-1}(S)$ on $E_{1}$, and inductively we may calculate $m(S, i)$.

Let $J$ be a coherent ideal of $Z$, and $\pi_{1} \circ \ldots \circ \pi_{n}$ be a chain of blowing-up. We define by induction $\pi^{* 0}(J)=J$, and for $i>0$

$$
\pi^{* \imath}(J)=\left(\pi_{i}^{*}\left(\pi^{*(i-1)}(J)\right):\left(I\left(Z_{i}, E(i, i)\right)\right)^{\delta(\imath)}\right)
$$

where $\delta(i)$ is the multiplicity of $\pi^{*(i-1)}(J)$ along the center of $\pi_{i}$, and $I\left(Z_{i},(E(i, i))\right.$ the ideal sheaf of $E(i, i)$ in $Z_{i}$.

LEMma 3.2. Let $\pi_{1} \circ \ldots \circ \pi_{n}: Z(n) \rightarrow Z$ be a good local blowingup. Provided we know

(i) The ordered tree of $\pi_{1} \circ \ldots \circ \pi_{r}(r \leq n)$

(ii) $\forall i \leq r$ the ideal $J_{i}$ such that $\pi^{*(i-1)}\left(J_{i}\right)$ define the center of $\pi_{i}$. Then we may explicitly calculate:

(a) $\forall i \leq r$ the divisor $D_{i}$ associated to $E(i, i)$ by $\pi_{\imath+1} \circ \ldots \circ \pi_{n}$

(b) $\forall j \leq i \leq r$ the divisor $D_{j, \imath}$ associated to $E(j, i)$ by $\pi_{i+1} \circ \ldots \circ \pi_{n}$

(c) For all surface $S$ of $Z, \forall i \leq r$ the divisor $D_{i} S$ associated to $S_{i}$ by $\pi_{i+1} \circ \ldots \circ \pi_{n}$.

Proof. We assume that $I(a)$ is the ideal sheaf of $E(a, a)$ in $Z(a)$ and $b(i, j)=$ multiplicity of $\pi^{*(\jmath-1)}\left(J_{\imath}\right)$ along the center of $\pi_{\jmath}$

(a) We may calculate inductively the divisors $D_{i}$ as follows:

$D_{1}$ is the divisor determined by $\pi^{*}\left(J_{1}\right)$ and the divisor determined by $\pi^{*}\left(J_{\imath}\right)$ is $\left[\sum_{\jmath<i} b(i, j) D_{\jmath}\right]+D_{i}$ because $\forall s, 0 \leq s \leq i$

$\left(\pi_{s+1} \circ \ldots \circ \pi_{n}\right)^{*}\left(\pi^{*_{s}}\left(J_{i}\right)\right)=\left(\pi_{s+2} \circ \ldots \circ \pi_{n}\right)^{*}\left(I(s+1)^{b(i, s+1)} \pi^{*_{s+1}}\left(J_{i}\right)\right)$

then:

$$
\begin{aligned}
\pi^{*}\left(J_{\imath}\right)= & {\left[\left(\pi_{2} \circ \ldots \circ \pi_{n}\right)^{*}\left(I(1)^{b(i, 1)}\right)\right] \bullet\left[\left(\pi_{3} \circ \ldots \circ \pi_{n}\right)^{*}\left(I(2)^{b(\imath, 2)}\right)\right] } \\
& \bullet \ldots \bullet\left[\left(\pi_{i} \circ \ldots \circ \pi_{n}\right)^{*}\left(I(i-1)^{b(i, \imath-1)}\right)\right] \bullet\left[\left(\pi_{i} \circ \ldots \circ \pi_{n}\right)^{*}\right. \\
& \left.\left(\pi^{*_{2}}\left(J_{i}\right)\right)\right] .
\end{aligned}
$$


(b) Let us prove these points by induction:

$$
\begin{aligned}
D_{j}^{j} & =\left(\pi_{j+1} \circ \ldots \circ \pi_{n}\right)^{-1}(E(j, j))=D_{j} \\
D_{\jmath}^{i-1} & =\left(\pi_{i} \circ \ldots \circ \pi_{n}\right)^{-1}(E(j, i-1)) \\
& =\left(\pi_{i+1} \circ \ldots \circ \pi_{n}\right)^{-1} \pi_{\imath}^{-1}(E(j, i)) \\
& =\left(\pi_{i+1} \circ \ldots \circ \pi_{n}\right)^{-1}(E(j, i+1))+a(j, i) E(i, i) \\
& =D_{j}^{i}+a(j, i) D_{i} .
\end{aligned}
$$

(c) It is enough to show inductively that

$$
D_{i} S=D_{Z(n)} \pi^{-1}(S)-\sum_{j \leq i} m(S, j) D j
$$

these formula is true because:

$$
\begin{aligned}
D_{0} S & =\pi^{-1}(S) \\
D_{\imath-1} S & =\left(\pi_{i} \circ \ldots \circ \pi_{n}\right)^{-1}\left(S_{\imath-1}\right)=\left(\pi_{\imath+1} \circ \ldots \circ \pi_{n}\right)^{-1}\left(\pi_{i}^{-1}\left(S_{\imath-1}\right)\right)= \\
& =\left(\pi_{i+1} \circ \ldots \circ \pi_{n}\right)^{-1}\left(m(S, i) E(i, i)+S_{i}\right)=m(S, i) D_{\imath}+D_{i} S
\end{aligned}
$$

and the formula follows.

Proposition 3.3. Let $\pi=\pi_{1} \circ \ldots \circ \pi_{n}: Z(n) \rightarrow Z(0)=Z$ be $a$ local blowing-up, if we know the ordered tree of $\pi_{1} \circ \ldots \circ \pi_{d}$, then we have $\forall i \leq d$ an ideal sheaf $J_{i}$ such that $\pi^{*_{2-1}}\left(J_{i}\right)$ is the ideal sheaf of the center of $\pi_{i}$.

Proof. By induction over $i$.

(a) For $i=1, J_{1}$ is the ideal of $\pi\left(E_{1}\right)$ in $Z$.

(b) Let $i>1$ and let us suppose that the assertion is true for $r<i$.

For all $r<i$ there are a finite set of generators of $J_{r}, G(r)$, such that $m(S, r)=1, m(S, t)=0 \forall S \in G(r), \forall t>r$.

If we construct a finite set $H(r)$ of surfaces of $Z$ such that:

(i) $\forall t, r \leq t \leq i, \forall S \in H(r), m(S, t)$ depends only on $t$.

(ii) The ideal sheaf of $Z(n)$ generated by the local equations of

$$
\left\{D_{Z(n)} S_{n}+D_{\imath} S: S \in H(r)\right\}
$$

is equal to the one generated by the local equations of

$$
\left\{D_{\eta(n)} T_{n}+D_{1} T: m(T, i)=1\right\} .
$$


Then one has that:

$$
\text { center of } \pi_{i}=\bigcap_{S \in H(r)} S_{i-1} \text {. }
$$

To get $H(r), \forall r$, we begin with a finite set $H(i)$ of surfaces of $Z$ which verifies (ii), then inductively $H(r)$ is obtained from $H(r+1)$ as follows: if

$$
\delta(r)=\max \{m(S, r): S \in H(r+1)\}
$$

then

$$
H(r)=\left\{S \cdot T^{(\delta(r)-m(S, r))}: S \in H(r+1), T \in G(r)\right\}
$$

With this construction $J_{i}$ is the ideal generated by $H(0)$.

REMARK 3.4. The assertion of Proposition 2.3 is also true for $\left\{\pi_{i}: 1 \leq i \leq d\right\} \bigcup\left\{\pi_{c}\right\}$ when we know:

(a) The ordered tree of $\pi_{1} \circ \ldots \circ \pi_{d}$

(b) $\forall i, i \leq d$ the arrows $\left(E_{i}, E_{c}\right)$

With the additional condition $\forall j, d<j<c,\left(E_{j}, E_{c}\right)$ is not an arrow. The proof is the same as Proposition 2.3.

THEOREM 3.5. Given a combinatorial local blowing-up $\pi$, we may determine the ordered tree of a local blowing-up $\pi^{\prime}$ equivalent to $\pi$.

Proof. Let $X, Y, T$ surfaces associated to $\pi$, and let $D_{X}=$ $\sum_{i \geq 1} a_{i, 1} E^{i}$, (resp. $D_{Y}=\sum_{i \geq 1} b_{i, 1} E_{i}, \quad D_{T}=\sum_{i \geq 1} c_{i, 1} E_{i}$ ) be the associated divisor to the surface $X$ (resp. $Y, T$ ). Then we may choose the first component $E_{i}$ by releasing an index $i$ in such a way that

(a) $\max \left\{a_{i, 1}, b_{i, 1}, c_{\imath, 1}\right\}=1$.

(b) $a_{i, 1}+b_{i, 1}+c_{\imath, 1}=3$, or $a_{i, 1}+b_{\imath, 1}+c_{i, 1}=2$ and $\pi^{-1}\left(\pi\left(E_{i}\right)\right)$ is a divisor.

In the first case $\pi_{1}$ is a quadratic transformation, in the second one is monoidal.

Let us call $A(1)$ the tree $\left\{E_{1}\right\}$ and let us suppose now that we know the tree $A(r)$ of $\pi_{1} \circ \ldots \circ \pi_{r}$. 
By Lemma 3.2 we know the divisors:

$$
\begin{aligned}
D_{r} X & =\sum_{i>r} a_{i, r+1} E_{i} \\
D_{r} Y & =\sum_{\imath>r} b_{i, r+1} E_{\imath} \\
D_{r} T & =\sum_{i>r} c_{\imath, r+1} E_{i} \\
D_{j}^{r} & =\sum_{i>r} s(j, r, i) E_{i} .
\end{aligned}
$$

Then $E_{d}$ is the $(r+1)$-th component if $d$ satisfies:

$$
\begin{aligned}
& \text { (a) } \max \left\{a_{i, r+1}, b_{i, r+1}, c_{i, r+1}, s(j, r, d): j \leq r\right\}=1 \\
& \text { (b) } a_{\imath, r+1}+b_{i, r+1}+c_{i, r+1}+\sum_{\jmath \leq r} s(j, r, d)=3
\end{aligned}
$$

( $\pi_{d}$ is a quadratic transformation); or

$$
a_{i, r+1}+b_{i, r+1}+c_{i, r+1}+\sum_{j \leq r} s(j, r, d)=2
$$

( $\pi_{d}$ is a monoidal transformation), and $\pi^{*}\left(J_{d}\right)$ is inversible, where $J_{d}$ is the ideal which corresponds by Remark 3.4 to the ordered tree $\left\{E_{i}: 1 \leq i \leq r\right\} \bigcup\left\{E_{d}\right\}$ with the arrow $\left(E_{i}, E_{d}\right)$ if $s(i, r, d)=$ 1.

\section{The general situation.}

THEOREM 4.1. Given a good local blowing-up $\pi$ we may determine the ordered tree of a local blowing-up $\pi^{\prime}$ equivalent to $\pi$; and the class (monoidal or quadratic) of each component.

Proof. Let $\sum_{i \in I(1)} E_{i}$, be the exceptional divisor of $\pi$, and if $\pi_{1}^{\prime} \circ$ $\ldots \circ \pi_{r}^{\prime}$ is determined, let $D_{r} S=\sum_{i \in I(r)} a^{r}(S, i) E_{i}$ the associated divisor to $S_{r}$ by $\pi$ (3.2.c).

Let us call $P(1)$ the quotient set $I(1) / \equiv$ by the relation:

$i \equiv j$ if for every surface $S$ of $Z$ is $a^{0}(S, i) \neq 0 \Leftrightarrow a^{0}(S, j) \neq 0$ this is equivalent to $i \equiv j$ if $\pi\left(E_{i}\right)=\pi\left(E_{j}\right)$. 
If $j^{\prime} \in P(1)$ let $A\left(1, j^{\prime}\right)=\left\{\imath \in I(1): i \in j^{\prime}\right\}$.

Let $j^{\prime} \in P(1)$ and $t \in A\left(1, j^{\prime}\right)$, by Th. $2.3 E_{t}$ will be the first element of $\left\{E_{\imath}: \imath \in A\left(1, j^{\prime}\right)\right\}$ if $\forall i \in A\left(1, j^{\prime}\right)$ is $a^{0}\left(S, E_{t}\right)<a^{0}\left(S, E_{i}\right)$, for every surface $S$ of $Z$ such that $Y_{j} \subset S$.

Let, $\forall b^{\prime} \in P(1), E_{1\left(b^{\prime}\right)}$ be the first element of $\left\{E_{i}: i \in A\left(1, b^{\prime}\right)\right\}$, then if $j^{\prime}$ verifies that :

(a) $\pi^{-1}\left(E_{1\left(\jmath^{\prime}\right)}\right)$ is a divisor.

(b) For all $k^{\prime}$, such that $\pi^{-1}\left(\pi\left(E_{1\left(k^{\prime}\right)}\right)\right)$ is a divisor, is $\pi\left(E_{1\left(k^{\prime}\right)}\right) \not \subset$ $\pi\left(E_{1\left(j^{\prime}\right)}\right)$.

Then $E_{1\left(\jmath^{\prime}\right)}$ will be the divisor corresponding to the first factor of $\pi^{\prime}$ and we will change this $j^{\prime}$ by $1^{\prime}$ and call $E_{1}=E_{1\left(1^{\prime}\right)}$.

Let us assume that we know the ordered tree of $\pi_{1}^{\prime} \circ \ldots \circ \pi_{v}^{\prime}$ and the sets $A\left(v, j^{\prime}\right), j^{\prime} \in P(v)$. Let $Q(v+1)$ the quotient set $\left(A\left(v, v^{\prime}\right)-\left\{v(v)^{\prime}\right\}\right) / \sim$ by the relation:

$i \sim j$ if for every surface $S$ of $Z$ is $a^{v}(S, i) \neq 0 \Leftrightarrow a^{v}(S, j) \neq 0$. Then $P(v+1)=\left(P(v)-\left\{v^{\prime}\right\}\right) \cup Q(v+1)$.

Let $j^{\prime} \in P(v+1), A\left(v+1, j^{\prime}\right)=\left\{i \in I(v+1): i \in j^{\prime}\right\}$. By Theorem $2.3 E_{(v+1)\left(j^{\prime}\right)}$ is the first element of $\left\{E_{\imath}: i \in A\left(v+1, j^{\prime}\right)\right\}$ if $\forall i \in A\left(v+1, j^{\prime}\right)$, and for every surface $S$ of $Z$ is $a^{v}\left(S,(v+1)\left(j^{\prime}\right)\right) \leq$ $a^{v}(S, i)$.

We extend the ordered tree of $\pi_{1}^{\prime} \circ \ldots \circ \pi_{v}^{\prime},\left\{E_{\imath}: 1 \leq i \leq v\right\}$ to $\left\{E_{(v+1)\left(\jmath^{\prime}\right)}: j^{\prime} \in P(v+1)\right\} \cup\left\{E_{i}: 1 \leq i \leq v\right\}$ by adding the arrows $\left(E_{i}, E_{(v+1)\left(j^{\prime}\right)}\right)$ if the multiplicity of $D_{i}^{v}$ at $E_{(v+1)\left(j^{\prime}\right)}$ is 1 .

By Remark 3.4 we assign to each $E_{(v+1)\left(j^{\prime}\right)}$ the ideal $J_{(v+1)\left(j^{\prime}\right)}$. There is at least one $E_{(v+1)\left(j^{\prime}\right)}$ such that

(a) $\pi^{*}\left(J_{(v+1)\left(j^{\prime}\right)}\right)$ is an invertible ideal of $Z^{\prime}$.

(b) $J_{(v+1)\left(k^{\prime}\right)} \not \subset J_{(v+1)\left(j^{\prime}\right)}, \forall k^{\prime} \neq j^{\prime}$.

Then we may take as $\pi_{v+1}^{\prime}$ the blowing-up corresponding to this $E_{(v+1)\left(\jmath^{\prime}\right)}$.

The codimension of center of each $\pi_{\imath}^{\prime}$ is calculated inductively by the following lemma (see [6]).

LEMma 4.2. [6]. Let $\left\{x_{1}, x_{2}, x_{3}\right\}$ be a local coordinate system on $(Z, \mathbf{p})$ centered at $\mathbf{p}$. Let $\pi=\pi_{1} \circ \ldots \circ \pi_{n}: Z_{n} \rightarrow Z$ be a local blowing$u p$, and $Y_{i}$ the center of $\pi_{i}(i=1, \ldots, n)$. If $D=\sum_{i<r} \beta_{i} E_{\imath(r-1)}$, is the divisor of $Z_{r-1}$ determined by $\left(\pi_{1} \circ \ldots \circ \pi_{r-1}\right)^{*}\left(d x_{1} \wedge d x_{2} \wedge d x_{3}\right)$ and $d$ is the codimension of $Y_{r}$, then the divisor of $Z(r)$ determined by $\left(\pi_{1} \circ \ldots \circ \pi_{r}\right)^{*}\left(d x_{1} \wedge d x_{2} \wedge d x_{3}\right)$ is: 


$$
\sum_{\imath<r} \beta_{i} E_{\imath}(r)+\left[d-1+\sum_{i<r} \beta_{\imath} \cdot \text { mult }_{D} E_{i(r-1)}\right] E_{r(r)} .
$$

5. Quasi-ordinary singularities. Let $S$ be a surface of $Z$, and $\mathbf{p}$ an quasi-ordinary singularity of $S$. We call canonical blowing-up of $(S, \mathbf{p})$ to a local blowing-up $(\pi)=\left(\pi_{1} \circ \ldots \circ \pi_{n}\right):\left(Z_{n}, \pi_{-1}(\mathbf{p})\right) \rightarrow$ $(Z, \mathbf{p})$ such that $\forall i, \quad 1 \leq i \leq n, \pi_{\imath}$ is permissible, i.e. if $S_{i-1}$ have permissible curves then $\pi_{\imath}$ is a monoidal transformation with permissible center for $S_{\imath-1}$.

We call canonical resolution of $(S, \mathbf{p})$ to a canonical local blowingup such that:

(a) $\left(S_{n}, \pi^{-1}(\mathbf{p})\right)$ is regular.

(b) $\left(S_{n}, \pi^{-1}(\mathbf{p})\right)$ have normal crossing with the components of exceptional divisor of $(\pi)$.

The set of normalized characteristic pairs of $(S, \mathbf{p})$ determine the canonical resolutions of $(S, \mathbf{p})$ (see $[\mathbf{3}])$. Moreover if $(\pi)$ is a canonical resolutions of $(S, \mathbf{p})$ we may know the ordered tree of every canonical resolutions equivalent to $(\pi)$, and the class of their factors. With this information we determine the first pair of $\left(S_{i}, \mathbf{p}_{\imath}\right)$, and the set of normalized characteristic pair of $(S, \mathbf{p})$ because:

If $\left(\pi \circ \pi^{\prime}\right)$ is a canonical local blowing-up of the quasi-ordinary singularity $(T, \mathbf{p})$, then:

(1) We know the formulaes which establish relation between the set of normalized characteristic pairs of strict transform of $(T, \mathbf{q})$, (see $[3])$.

(2) The transform of the first characteristic pair is eqivalent to $(1 / m, 0),(m \in \mathbb{N})$, if and only if $\pi^{\prime}$ is monoidal and the multiplicity of $T$ along the center of $\pi$ is multiple of the multiplicity of strict transform of $T$ along the center of $\pi^{\prime}$.

\section{REFERENCES}

[1] J. M. Aroca, H. Hironaka and J. L. Vicente, The theory of the maximal contact, Memorias de Matemática del Instituto Jorge Juan, $n^{0} 29$ C.S.I.C. Madrid 1976.

[2] V. Cossart, Polièdre charactéristique et éclatements combinatoires, Rev. Mat. Iberoamericana, $5 n^{0} 1,2$, (1989).

[3] J. Lipman, Quasi-ordinary Singularities of Embedded Surfaces, Ph. D. Thesis. Harvard University, 1965.

[4] Quasi-ordinary singularities of surfaces in $C^{3}$, in Proc. Sympos. Pure Math., 40, part 2. 
[5] I. Luengo Velasco, On the structure of embedded algebroid surfaces, Proc. Sympos. Pure Math., 40, part 2, 1983.

[6] W. Veys, Relations between numerical data of an embedded resolution, Amer. J. Math., 113 (1991), 573-592.

Received October 14, 1992.

UNIVERSIDAD DE VALLADOLID

47005 VALladolid (SPAIN) 




\section{PACIFIC JOURNAL OF MATHEMATICS}

Volume $167 \quad$ No. $2 \quad$ February 1995

Existence of shortest directed networks in $\mathbb{R}^{2}$

201

MANUEL AlFARO GARCIA

Hecke characters of singular Drinfel'd modules

215

SUNGHAN BAE

Factorization method for a bimeromorphic morphism

231

Jose PEREZ BLANCO

$L^{p}$ estimates for operators associated to flat curves without the Fourier 243 transform

Anthony Carbery, James Thomas Vance, JR., Stephen

WAINGER, DAVID K. WATSON and JAMES WRIGHT

$S$-integer points on elliptic curves

263

ROBERT HOWARD GROSS and JOSEPH SILVERMAN

On metrics defined by modules

JAMES ALLISTER JENKINS

Conditional Wiener integrals. II

ChUll PARK and DAVID LeE SKOUG

On a Plancherel formula for certain discrete, finitely generated, torsion-free nilpotent groups

CAROLYN PFEFFER JOHNSTON

Desingularizations of some unstable orbit closures

MARK STEPHEN REEDER

Determining multiplicities of half-integral weight newforms

Thomas Richard SHEMANSKE and LyNne WALLING

Generation of integral orthogonal groups over dyadic local fields 